\title{
Shockwave treatment of erectile dysfunction
}

\author{
Ilan Gruenwald, Boaz Appel, Noam D. Kitrey and Yoram Vardi
}

\begin{abstract}
Low-intensity extracorporeal shock wave therapy (LI-ESWT) is a novel modality that has recently been developed for treating erectile dysfunction (ED). Unlike other current treatment options for ED, all of which are palliative in nature, LI-ESWT is unique in that it aims to restore the erectile mechanism in order to enable natural or spontaneous erections. Results from basic science experiments have provided evidence that LI-ESWT induces cellular microtrauma, which in turn stimulates the release of angiogenic factors and the subsequent neovascularization of the treated tissue. Extracorporeal shock wave therapy (ESWT) has been clinically investigated and applied in several medical fields with various degrees of success. High-intensity shock wave therapy is used for lithotripsy because of its focused mechanical destructive nature, and medium-intensity shock waves have been shown to have anti-inflammatory properties and are used for treating a wide array of orthopedic conditions, such as non-union fractures, tendonitis, and bursitis. In contrast, LI-ESWT has angiogenetic properties and is therefore used in the management of chronic wounds, peripheral neuropathy, and in cardiac neovascularization. As a result of these characteristics we initiated a series of experiments evaluating the effect of LI-ESWT on the cavernosal tissue of patients with vasculogenic ED. The results of our studies, which also included a double-blind randomized control trial, confirm that LI-ESWT generates a significant clinical improvement of erectile function and a significant improvement in penile hemodynamics without any adverse effects. Although further extensive research is needed, LI-ESWT may create a new standard of care for men with vasculogenic ED.
\end{abstract}

Keywords: erectile dysfunction, male impotence, shockwaves, therapy

\section{Introduction}

The current nonsurgical treatment modalities in the management of erectile dysfunction (ED) mainly consist of oral phosphodiesterase type 5 inhibitors (PDE5is) and/or intracavernosal injections of vasodilating agents. These treatments are very effective and are reasonably safe with rare unwanted or adverse effects. However, they all share the same major drawback: they do not alter the underlying pathophysiology of the erectile mechanism. These treatments are usually taken on demand, prior to the sexual act, and their effect is essentially time limited. Although daily administration of a PDE5i instead of on-demand treatment does address some of these problems, it still does not modify the pathophysiology of the erectile process. Moreover, the evidence that its effect on the erectile tissue is long-lasting is very limited. Presently, only a small number of men with ED can be offered treatment that would restore their spontaneous erectile function. This group includes those who would benefit from various lifestyle or drug regimen modifications, those who can be treated for relevant endocrine disorders, or those with vasculogenic $\mathrm{ED}$ who would benefit from microvascular surgery. Most patients with ED rely on their treatment in order to maintain their sexual function; providing a treatment for men with ED that is rehabilitative or even curative and enables them to regain spontaneous sexual activity with normal intimacy and without adverse effects is an unmet medical goal. Recently, data from several studies have accumulated that this goal could probably be met by low-intensity extracorporeal shockwave therapy (LI-ESWT) of the corpora cavernosa. This review intends to summarize the scientific background underlying the effect of this energy as well as recent clinical evidence of its effect in patients with vasculogenic ED.

\section{Background}

Shockwaves (SWs) are acoustic waves that carry energy and when propagating through a medium,
Ther Adv Urol

(2013) 5(2) 95-99

DOI: $10.1177 /$

1756287212470696

(c) The Author(s), 2013. Reprints and permissions: http://www.sagepub.co.uk/ journalsPermissions.nav

Correspondence to: Ilan Gruenwald, MD Neuro-Urology Unit, Rambam Medical Center and Rappaport Faculty of Medicine, Technion, Haifa 34679, Israel I_gruenwaldarambam. health.gov.il

Boaz Appel, MD Noam D. Kitrey, MD Yoram Vardi, MD, PhD Neuro-Urology Unit, Rambam Medical Center and Rappaport Faculty of Medicine, Technion, Haifa Israel 
can be targeted and focused noninvasively to affect a distant selected anatomical region.

When LI-ESWT is applied to an organ, the relatively weak yet focused SWs interact with the targeted deep tissues where they cause mechanical stress and microtrauma. This stress and microtrauma (also known as shear stress) induces a cascade of biological reactions that result in the release of angiogenic factors which in turn triggers neovascularization of the tissue with subsequent improvement of the blood supply.

\section{LI-ESWT in vitro and animal studies}

Research on the biological effects of LI-ESWT has mainly been focused on vasculogenesis and local neovascularization. Wang and colleagues [Wang et al. 2003] discovered that LI-ESWT stimulates the expression of angiogenesis-related growth factors, such as endothelial nitric oxide synthase (eNOS) and vascular endothelial growth factor (VEGF), and endothelial cell proliferation factors, such as proliferating cell nuclear antigen (PCNA). They also reported that LI-ESWT induces neovascularization, and consequently improves blood supply. Interestingly, they found that 1 week after LI-ESWT, the angiogenic marker levels rose significantly and this effect lasted for approximately 8 weeks. They also showed that neovascularization and cell proliferation were evident 4 weeks after LI-ESWT and persisted for more than 12 weeks. The same group [Wang et al. 2003] investigated the effect of LI-ESWT on neovascularization of the tendon-bone junction. For this purpose, LI-ESWT was applied to the Achilles tendon junction of 50 New Zealand rabbits. The extent of neovascularization was determined from the expression of VEGF, eNOS, and PCNA. They found that the number of neovessels and the expressions of the angiogenic markers and PCNA were substantially increased by LI-ESWT. This group previously reported similar findings in a smaller canine study [Wang et al. 2002] on the effect of this energy on bone-tendon junction in eight dogs: new capillaries and muscularized vessels were seen in obtained specimens 4 and 8 weeks after local LI-ESWT, with no change in the untreated sites.

The effect of LI-ESWT on intracellular VEGF levels has also been reported by Gutersohn and colleagues [Gutersohn et al. 1999] in human umbilical vein endothelial cells (HUVECs). They found that levels of VEGF mRNA in the LI-ESWT-treated cells were significantly greater than those in the untreated controls. The effect of LI-ESWT on intracellular VEGF levels in HUVECs has also been reported by Nishida and colleagues [Nishida et al. 2004], who found that LI-ESWT significantly increased the expression of VEGF mRNA and its receptor, Flt-1. Their investigations on the effects of LI-ESWT on a porcine model of chronic myocardial ischemia also showed that VEGF expression was significantly upregulated in the ischemic myocardial cells after treatment [Nishida et al. 2004].

Progenitor cell therapy has recently been suggested as a new approach to boost neovascularization of ischemic tissues. During acute ischemia, the release of chemo-attractant factors (i.e.VEGF) act as homing factors for circulating progenitor cells (CPCs). Aicher and colleagues [Aicher et al. 2006] investigated the effect of LI-ESWT on homing of infused human CPCs in rats with chronic hind limb ischemia. For this purpose, they applied LI-ESWT (500 hits) to the adductor muscles of the right hind limb of rats (the left hind limbs were used as the controls). Twenty-four hours after LI-ESWT, labeled CPCs were then injected. Forty-eight hours following labeled human CPC injection to the rats. They found a substantially higher number of CPCs in the SW-treated versus the untreated adductor muscles. A significant increase in blood flow was also documented following CPC treatment and LI-ESWT. From these results, Aicher and colleagues concluded that LI-ESWT may improve the efficacy of CPC treatment in chronic ischemia.

\section{LI-ESWT for cardiac disease}

The effect of LI-ESWT on the myocardium has also been intensively studied in recent years. In a porcine model of ischemia-induced myocardial dysfunction, Nishida and colleagues [Nishida et al. 2004] applied LI-ESWT to chronic ischemic hearts of 28 domestic pigs. They found that LI-ESWT improved regional myocardial blood flow and the wall thickening fraction, and even brought about complete recovery of the left ventricular (LV) ejection fraction. In contrast, sustained myocardial dysfunction was found in the pigs which did not receive LI-ESWT. No complications, including arrhythmias, were observed during or after the treatment. In another study in pigs with an acute myocardial infarction, Uwatoku and colleagues [Uwatoku et al. 2007] demonstrated that LI-ESWT has a positive effect on LV remodeling. Finally, Ito and colleagues [Ito et al. 2010] showed that LI-ESWT also improved LV remodeling after the myocardial ischemiareperfusion injury. 
Clinically, the effect of LI-ESWT on the heart has also been investigated in a double-blind shamcontrolled study in eight human patients with severe ischemic heart disease [Kikuchi et al. 2010]. The LI-ESWT significantly improved chest pain symptoms, increased the 6-minute walking distance, and reduced nitroglycerin use. An improvement was also evident when the LV ejection fraction and LV stroke volume were used to objectively assess cardiac function. Importantly, they reported that LI-ESWT was safe without any complications or adverse effects.

Yang and colleagues [Yang et al. 2012], in a randomized, double-blind, controlled study, also investigated the effects of LI-ESWT in 25 patients with ischemic heart disease in which angina severity scales and questionnaires were used to measure the response. Their results were similar to those that were reported by Kikuchi and colleagues [Kikuchi et al, 2010]. None of the patients in the control group reported improvements after treatment. Comparable results have also been reported by Vasyuk and colleagues [Vasyuk et al. 2010] and Wang and colleagues [Wang et al. 2012] in patients with severe coronary artery disease and refractory angina to whom LI-EWST was applied.

\section{LI-ESWT for ED}

Since one of the underlying functional causes of ED is poor cavernosal arterial blood flow, we hypothesized that inducing neovascularization by LI-ESWT could potentially improve cavernosal arterial flow which in turn would improve erectile function. If this hypothesis could be proved, LI-ESWT could then become an effective and noninvasive treatment for ED.

The purpose of our first study was to evaluate the feasibility, efficacy, and safety of LI-ESWT in 20 men, aged $56.1 \pm 10.7$ years, with mild to moderate $\mathrm{ED}$ due to cardiovascular disease and without any neurogenic etiology [Vardi et al. 2010]. These patients had ED for almost 3 years (average), and all were able to function sexually with the use of PDE5i (i.e. PDE5i responders). Our treatment protocol was based on the described methodology used in cardiac LI-ESWT [Kikuchi et al. 2010], with modifications according to the depth of the target tissue (corpora) and to anatomical differences. We applied 300 SWs (energy intensity of $0.09 \mathrm{~mJ} / \mathrm{mm}^{2}$ ) to each of five different sites: three along the penile shaft and two at the crural level. The protocol consisted of two treatment sessions per week for 3 weeks, a 3-week no-treatment interval, and a second 3-week treatment period of two treatment sessions per week.

One month after LI-ESWT, the erectile function in 15 men improved. An increase by more than five points in the International Index of Erectile Function - Erectile Function (IIEF-EF) domain score was noted in 14 men, and by more than 10 points in 7 men. Five men did not respond to LI-ESWT. Overall, the average increase in the IIEF-EF domain scores was 7.4 points (13.5$20.9, p=0.001)$. Furthermore, erectile function and penile blood flow were measured using nocturnal penile tumescence (NPT) and venous occlusion plethysmography of the penis, respectively. LI-ESWT improved all NPT parameters, especially in the 15 men who responded to LI-ESWT, where significant increases in the duration of the erections and penile rigidity were recorded. Penile blood flow also improved significantly and a strong correlation was found between the increase in the IIEF-EF domain scores and the improvement in penile blood flow at the 1-month follow-up examination. At the 6-month follow-up visit, 10 men reported that they still had spontaneous erections that were sufficient for penetration and did not require PDE5i support.

In view of these very successful preliminary results, the effect of LI-ESWT was further investigated in a group of men whose ED was more severe than that of the first group of study patients [Gruenwald et al. 2012]. The average initial IIEF-EF domain score of the 29 men who were recruited for this second study was $8.8 \pm 1$. All 29 men had not responded to oral PDE5i therapy, and had multiple cardiovascular risk factors (23), cardiovascular disease (11), and diabetes mellitus (14). The specific aim of this second study was to investigate the ability of LI-ESWT to convert nonresponders to PDE5i therapy to PDE5i responders, so that they were able to achieve vaginal penetration with oral PDE5i therapy. The results were comparable to the first study. Three months after the completion of the LI-ESWT protocol, the IIEF-EF domain scores improved by at least five points in 22 men $(76 \%)$ and the mean IIEF-EF domain score increased by 10 points (to $18.8 \pm 1, p<0.0001$ ). At the end of the study, eight men (28\%) achieved normal erections (IIEF-EF domain score greater than 25) and 21 of the 29 men were able to achieve vaginal penetration with oral PDE5i therapy. Overall, 21 men $(72 \%)$ were converted to PDE5i responders. Cavernosal blood flow and penile endothelial function, as measured again by venous occlusion 
Table 1. The clinical studies included in the paper.

Gruenwald, I., Appel, B. and Vardi, Y. (2012) Low-intensity extracorporeal shock wave therapy - a novel effective treatment for erectile dysfunction in severe ED patients who respond poorly to PDE5 inhibitor therapy. J Sexual Med 9: 259-264.

Vardi, Y., Appel, B., Kilchevsky, A. and Gruenwald, I. (2012) Does low intensity extracorporeal shock wave therapy have a physiological effect on erectile function? Short-term results of a randomized, double-blind, sham controlled study. J Urol 187: 1769-1775.

Vardi, Y., Appel, B., Jacob, G., Massarwi, O. and Gruenwald, I. (2010) Can low-intensity extracorporeal shockwave therapy improve erectile function? A 6-month follow-up pilot study in patients with organic erectile dysfunction. Eur Urol 58: 243-248.

plethysmography of the penis (flow-mediated dilatation techniques [FMDs]), were both found to be significantly improved $(p=0.0001)$ in the men who responded to LI-ESWT.

In both studies, a strong and significant correlation between the subjective assessment of sexual function using validated sexual function questionnaires and the objective results of penile blood flow and erectile function was found. Moreover, none of the men in both studies reported treatmentassociated pain or any adverse events during or after the treatment.

The encouraging results from these two studies led us to conduct a prospective, randomized, doubleblind, sham-controlled study on 60 men with ED [Vardi et al. 2012]. In this study, we investigated the effects of LI-ESWT on erectile function and penile blood flow using the identical treatment protocol and study parameters that were used in our previous two studies. For the sham-treatment, we used a probe which did not produce any SW energy but looked identical to the treatment probe and produced the same noise and feeling of a 'hit'. The demographic characteristics and the baseline mean IIEF-EF scores of the treated and shamtreated patients of this third study were similar. We found that mean IIEF-EF domain scores of the treated men were significantly higher than those of the sham-treated men. This increase in the IIEF-EF domain scores was also accompanied by improvements in cavernosal blood flows and penile endothelial function, as measured by venous occlusion plethysmography of the penis (FMD). We have been following most of these men for more than 2 years and they all report that the beneficial response that was achieved immediately after therapy has not waned (Table 1).

\section{Discussion}

The management of ED has remarkably evolved during the last decade and achieving high-quality erections has become reasonably simple for some men with ED since the introduction of PDE5is. Nevertheless, all current available treatment modalities for ED are basically 'on-demand' therapies and their mechanism of action is to improve a single sexual encounter. One of the main research goals of this coming decade is finding a cure for ED. The current lines of investigation into new ED therapies are based on the Rho-kinase pathway, as well as exploring the feasibility of gene therapy through intracorporeal injections of plasmids and stem cell regenerative therapy. The introduction of a new therapeutic modality for ED whose underlying mechanism of action is unclear or unproven certainly warrants skepticism and criticism. Hence, there are more questions than answers regarding the therapeutic use of LI-ESWT for ED. On the other hand, our consistent and repeatable results withstand these doubts because the results from our three different studies not only confirm each other, but also demonstrate that LI-ESWT has a genuine physiological effect on the erectile mechanism. Although our results are promising, they are still limited. More doubleblind, randomized, controlled trials and long-term follow-up studies to confirm our findings are essential. There is also still much to investigate about the effect of LI-ESWT on the various types of ED, and the clinical parameters that could be used to predict who would benefit from LI-ESWT and who would not still require clarification, definition, and validation. There is also a need to determine the treatment protocols of LI-ESWT in order to establish the optimal protocol, in which the number of treatments and the number of penile sites to expose to LI-ESWT are defined. In this regard, we are already investigating different protocols and are offering a second 9-week treatment course for those who responded only partially to the first treatment course. Other studies are crucial for determining the optimal treatment protocol that will provide the best clinical outcome. Basic research is unquestionably required in order to explore and understand the mechanism of action of LI-ESWT on erectile tissue, as well as on other biologic systems. 


\section{Conclusions}

LI-ESWT is a revolutionary treatment of ED, and probably possesses unprecedented qualities that can rehabilitate erectile tissue. The clinical improvement in subjective erectile function together with the significant improvement in penile hemodynamics following LI-ESWT confirm that LI-ESWT has unique properties that may create a new standard of care for men with ED. LI-ESWT is both feasible and tolerable and without any adverse or unwanted effects. Its main advantage is its ability to improve and potentially restore erectile function in men with ED without additional pharmacotherapy. Hence, LI-ESWT is an appealing addition to the armamentarium of existing treatment options for $\mathrm{ED}$. In the near future we hope that LI-ESWT will be used for the long-term clinical management of ED either as an alternative or as an enhancer to the current treatments of ED.

\section{Acknowledgement}

The authors wish to thank Dr Arieh Bomzon, ConsulWrite (http://www.consulwrite.com), for his editorial assistance in preparing the manuscript.

\section{Funding}

This research received no specific grant from any funding agency in the public, commercial, or notfor-profit sectors.

\section{Conflict of interest statement}

ProfessorY.Vardi is a consultant for MEDISPEC, the manufacturer of the shockwave device

\section{References}

Aicher, A., Heeschen, C., Sasaki, K., Urbich, C., Zeiher, A. and Dimmeler, S. (2006) Low-energy shock wave for enhancing recruitment of endothelial progenitor cells: a new modality to increase efficacy of cell therapy in chronic hind limb ischemia. Circulation 114: 2823-2830.

Gruenwald, I., Appel, B. and Vardi, Y. (2012) Lowintensity extracorporeal shock wave therapy - a novel effective treatment for erectile dysfunction in severe ED patients who respond poorly to PDE5 inhibitor therapy. F Sexual Med 9: 259-264.

Gutersohn, A., Caspari, G., Vopahl, M. and Erbel, R. (1999) Upregulation of VEGF mRNA in HUVEC via shock Waves. In Proceedings of the International Conference From Genes to Therapy in Ischemic and Heart Muscle Disease, Marburg, Germany, 9-10 October.
Ito, Y., Ito, K., Shiroto, T., Tsuburaya, R., Yi, G., Takeda, M. et al. (2010) Cardiac shock wave therapy ameliorates left ventricular remodeling after myocardial ischemia-reperfusion injury in pigs in vivo. Coron Artery Dis 21: 304-311.

Kikuchi, Y., Ito, K., Ito, Y., Shiroto, T., Tsuburaya, R., Aizawa, K. et al. (2010) Double-blind and placebocontrolled study of the effectiveness and safety of extracorporeal cardiac shock wave therapy for severe angina pectoris. Circulat f 74: 589-591.

Nishida, T., Shimokawa, H., Oi, K., Tatewaki, H., Uwatoku, T., Abe, T. et al. (2004) Extracorporeal cardiac shock wave therapy markedly ameliorates ischemia-induced myocardial dysfunction in pigs in vivo. Circulation 110: 3055-3061.

Uwatoku, T., Ito, K., Abe, K., Oi, K., Hizume, T., Sunagawa, K. et al. (2007) Extracorporeal cardiac shock wave therapy improves left ventricular remodeling after acute myocardial infarction in pigs. Coron Artery Dis 18: 397-404.

Vardi, Y., Appel, B., Jacob, G., Massarwi, O. and Gruenwald, I. (2010) Can low-intensity extracorporeal shockwave therapy improve erectile function? A 6-month follow-up pilot study in patients with organic erectile dysfunction. Eur Urol 58: 243-248.

Vardi, Y., Appel, B., Kilchevsky, A. and Gruenwald, I. (2012) Does low intensity extracorporeal shock wave therapy have a physiological effect on erectile function? Short-term results of a randomized, double-blind, sham controlled study. F Urol 187: 1769-1775.

Vasyuk, Y., Hadzegova, A., Shkolnik, E., Kopeleva, M., Krikunova, O., Iouchtchouk, E. et al. (2010) Initial clinical experience with extracorporeal shock wave therapy in treatment of ischemic heart failure. Congestive Heart Failure 16: 226-230.

Wang, C., Huang, H. and Pai, C. (2002) Shock wave-enhanced neovascularization at the tendon-bone junction: an experiment in dogs. F Foot Ankle Surg 41: 16-22.

Wang, C., Wang, F., Yang, K., Weng, L., Hsu, C., Huang, C. et al. (2003) Shock wave therapy induces neovascularization at the tendon-bone junction. A study in rabbits. F Orthop Res 21: 984-989.

Wang, Y., Guo, T., Ma, T., Cai, H., Tao, S., Peng, Y. et al. (2012) A modified regimen of extracorporeal cardiac shock wave therapy for treatment of coronary artery disease. Cardiovasc Ultrasound 10: 35 .

Yang, P., Guo, T., Wang, W., Peng, Y., Wang, Y., Zhou, P. et al. (2012) Randomized and double-blind controlled clinical trial of extracorporeal cardiac shock wave therapy for coronary heart disease. Heart Vessels, in press.
Visit SAGE journals online http://tau.sagepub.com

(3)SAGE journals 